\title{
Dos nuevas especies del género Argyrana (Lepidoptera: Noctuidae)
}

\author{
Tania Olivares ${ }^{1} \&$ Andrés Angulo ${ }^{2}$ \\ 1. Casilla 4040 correo 3. Concepción, Chile; tolivare@udec.cl \\ 2. Universidad de Concepción, Facultad de Ciencias Naturales y Oceanográficas, Departamento de Zoología. Casilla \\ 160-C. Concepción, Chile; aangulo@udec.cl
}

Recibido 27-VII-2009. Corregido 19-IX-2009. Aceptado 21-X-2009.

\begin{abstract}
Two new species of the genus Argyrana (Lepidoptera: Noctuidae). The moth genus Argyrana was described by Koehler in 1951. We studied 539 specimens and here redescribe the adults, male and female genitalia. Two new species are reported for Araucanía and Aysén (IX Region and XI Region, Chile; the genus was previously known only from Argentina). These moths fly in October, November and February. Rev. Biol. Trop. 58 (1): 265-271. Epub 2010 March 01.
\end{abstract}

Key words: Lepidoptera, Argyrana, Cucullinae, new species, Andean-patagonian.

Argyrana es un género de "polilla" descrito por Koehler en 1951, basado en una sola especie, A. excellens. Se estudió el material tipo de la Colección y Fundación Miguel Lillo (IFML), Tucumán, Argentina; del Museo de Historia Natural de Munich, Alemania (ZMS) y de las colecciones Científicas de la Universidad de Concepción, Chile (UCCC) y se revisaron los géneros y especies descritos para la subfamilia Cucullinae por Koehler en su trabajo de 1951. Tras ello se encontraron tipos etiquetados de la especie Argyrana australis Koehler, la cual nunca fue descrita y publicada (nomen museologicum). Dicho material fue etiquetado por Koehler como Holotipo para un individuo castaño-lechoso y Alotipo para un individuo gris, las cuales son descritas como especies nuevas.

En Termas de Río Blanco y en Aysén (Chile) se recolectaron ejemplares machos y hembras de las dos formas de A. australis.

Este trabajo tiene como objetivo describir los adultos y los órganos genitales de estas especies y redescribir el género tanto los caracteres de los adultos como los órganos genitales.

\section{MATERIALES Y MÉTODOS}

Se revisaron un total de 539 ejemplares de los cuales 474 corresponden a la Región de Aysén (entre Cochrane y Villa OHiggins) $\left(45^{\circ} 30^{\prime} \mathrm{S}, 73^{\circ} 30^{\prime} \mathrm{W}\right)$ (27-379m.s.n.m.) y 65 ejemplares de la Región de la Araucanía

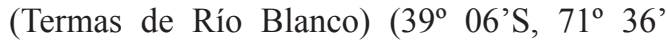
W) (865m.s.n.m.) de los cuales 24 ejemplares pertenecen al género Argyrana; luego de la revisión de la literatura especializada, principalmente Poole, 1989 que corresponde al catálogo mundial de nóctuidos, se estudiaron los tipos de la Colección y Fundación Miguel Lillo (IFML), Tucumán, Argentina; del Museo de Historia Natural de Munich, Alemania (ZMS) y también se incluyó material proveniente de las colecciones científicas de la Universidad de Concepción (UCCC) de Chile, en donde se depositaron los tipos de las especies descritas aquí. Mediante la técnica de disección de los órganos genitales de Angulo \& Weigert (1977) se procedió a realizar preparaciones microscópicas para la descripción de los órganos genitales del material recolectado y realizar las 
descripciones de los adultos. Las fotografías se realizaron con una cámara Panasonic Lumix DMC-FX3 y los dibujos lineales fueron realizados con una lupa estereoscópica con un brazo lateral incluido para el dibujo.

\section{RESULTADOS}

\section{Sufamilia Cucullinae \\ Argyrana Koehler, 1951}

Especie tipo: Argyrana excellens Koehler, 1951.

Diagnosis: alas anteriores grisáceas y castañoclaras, patagias y tégulas con escamas piliformes blanquizcas y castaño-claras, antenas plumosas aserradas, alas posteriores castañoclaras y blanquizcas con presencia de lúnula, banda subterminal notoria, dentada, delimitada por escamas negruzcas; expansión alar entre 31-34mm de promedio. Órganos genitales: uncus único, valvas oblongas, su largo es dos veces el ancho máximo, sin corona, ampulla presente, pequeña, terminada en punta, ubicada en el tercio distal de la valva, saccus triangular, aedeagus con presencia de espinas de base bulbosa y espinas delgadas, hembra con la bursa copulatrix unisacular.

Argyrana Koehler se diferencia de su género más próximo Albirenia Angulo \& Olivares por sus valvas alargadas, presencia de una ampulla única en la porción proximal de la valva y hembra con bursa copulatrix bisacular.

\section{Argyrana excellens Koehler, 1951}

(Figs. 1 y 6)

Macho-Hembra: Koehler, 1951. (descripción)

Genitales masculinos (Fig. 6): valvas oblongas, su largo es tres veces su ancho máximo, ampulla presente, pequeña, ubicada en la porción distal de la valva, sin corona.

Distribución geográfica: Argentina: Santa Cruz, Comodoro Rivadavia y Chubut.
Época de vuelo: Noviembre (Koehler 1951). Febrero (IFML, ZSM data).

Material examinado: 1 Fototipo macho, Cotypus, Com. Riv.-27, 21-II-1938, V.O., 1091, Argyrana excellens K (gen. prep.) (IFML); 1 Fototipo macho, Cotypus, Com. Riv.-27, 21-II1938, V. O., 1091, Argyrana excellens K, V. Oort Leg. (ZSM)

\section{Argyrana paulokehleri n. sp}

(Figs. 2-3 7, 9 y 11)

\section{ENGLISH DIAGNOSIS OF NEW TAXON}

Diagnosis: head with white and black scales, palps with brownish and whitish mixed scales, with piliform scales in the outer border, inner border with white piliform scales, antennae biserrate, eyes ciliate, vertex with whitish, blackish and with scales and the black line across, tegulae and patagiae covered with white mixed with dark brown and brownish scales and piliform scales. Forewings covered with white scales and brownish mixed scales, without macula and transversal line, only subterminal line is remarkable; hindwings covered with white and brownish mixed scales; genitalia: uncus rounded, with an apical spine, short valvae, without corona, triangle like saccus, short ampulla slightly curved, apex ampulla reach to the border of valva, cornuti with six thick spine of bulbous base.

Macho (Fig. 2): cabeza con escamas blancas y negras, palpos con escamas castaño claras y blanquecinas entremezcladas y con escamas piliformes en el borde externo, internamente con escamas piliformes blancas, antenas plumosas (aserradas), ojos con cilias distribuídas uniformemente, en la parte dorsal con escamas blancas; vertex con escamas blanquizcas, negruzcas y blancas, las escamas negras forman una línea que lo atraviesa, tégulas y patagias cubiertas por escamas y escamas piliformes blancas mezcladas con algunas escamas castaño oscuras y claras. Alas anteriores cubiertas por un color de fondo formado por escamas blancas mezcladas 

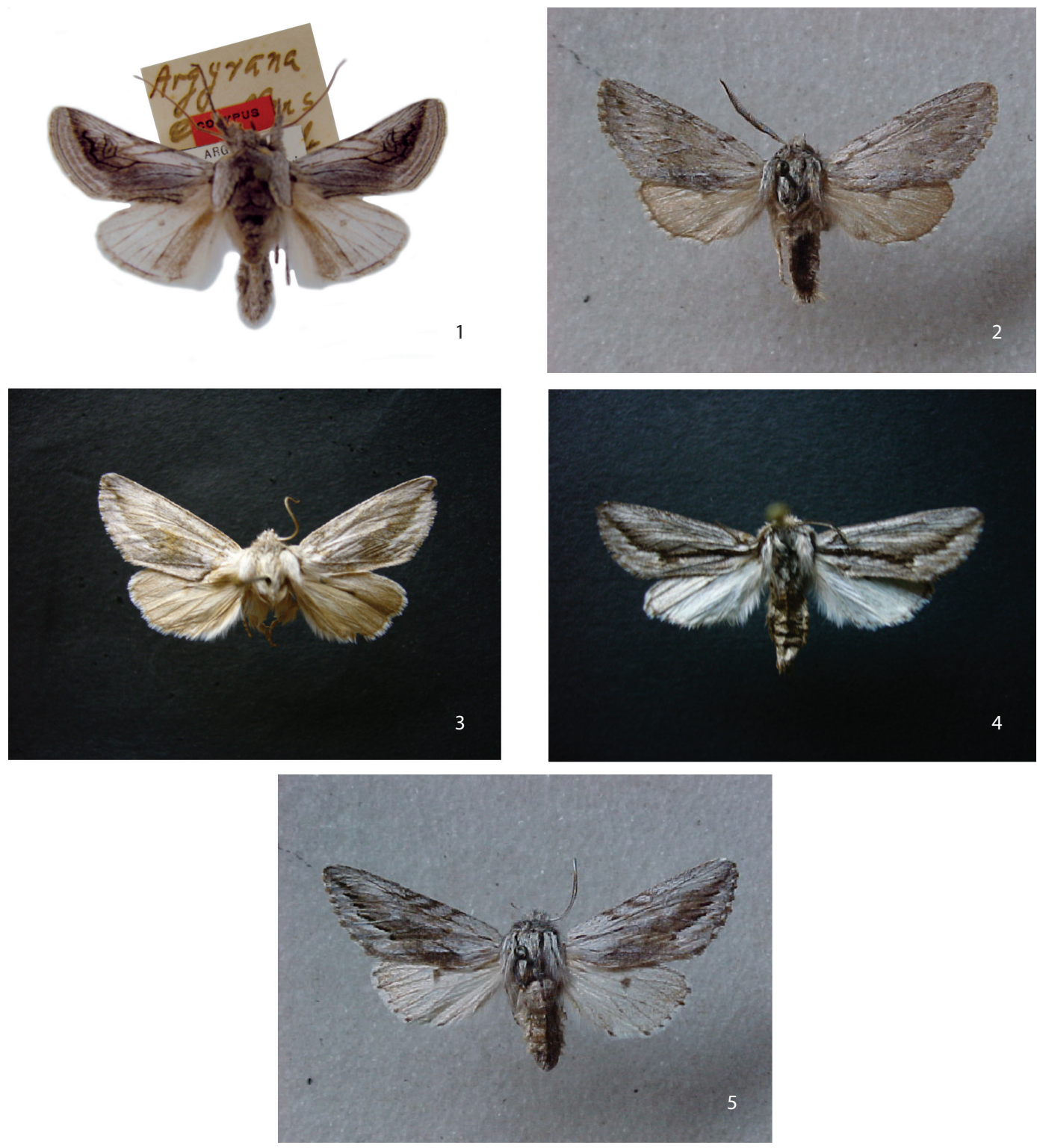

Fig. 1. Adulto Fototipo Argyrana excellens Koehler (ZSM); Fig. 2. Argyrana paulokehleri macho; Fig. 3. Argyrana paulokehleri hembra; Fig. 4. Argyrana bimorpha macho morfo; 1; Fig. 5. Argyrana bimorpha macho morfo 2.

Fig. 1. Adult Argyrana excellens Koehler (ZSM); Fig. 2. Argyrana paulokehleri male; Fig. 3. Argyrana paulokehleri female; Fig. 4. Argyrana bimorpha male morph; 1; Fig. 5. Argyrana bimorpha male morph 2. 
uniformemente con escamas castaño claras, sin manchas ni bandas notables, sólo la banda subterminal es notable, compuesta por una banda doble de escamas castaño-claras internamente y blancas en la parte externa; toda la banda es transversal y dentada; en el borde superior del ala en el sitio de la banda antemediana se ve una pequeña mancha de escamas castañas algo más oscuras que el color de fondo, concentradas; faz inferior cubierta por escamas normales y filiformes castaño claras iridiscentes, que le da una apariencia de dorado.

Alas posteriores cubiertas por escamas blancas y castaño-claras entremezcladas uniformemente, discal transparentada y castaño-clara, faz inferior con escamas blancas mezcladas con algunas castaño-claras; discal circular y cubiertas por escamas castaño-oscuras. Abdomen cubierto por escamas castaño-oscuras y blancas entremezcladas.

Genitales masculinos (Figs. 7 y 9): uncus redondeado, terminado en una espina, valvas cortas, sin corona, redondeadas en el ápice, ausencia de corona, saccus triangular, ampulla corta, levemente curvada, ubicada en el tercio distal, ápice de la ampulla llega al borde costal de la valva, cornuti con seis espinas gruesas, de base bulbosa, y microespinas.

Hembra (Fig. 3): similar al macho, con antenas ciliadas, cilias reducidas a la mitad del ancho máximo del segmento.

Genitales de la hembra (Fig. 11): apófisis anteriores subiguales en tamaño a las posteriores, bursa copulatrix unisacular, corpus bursae subtriangular, sin signa, ductus bursae poco esclerosado.

Distribución geográfica: Chile: IX Región: Prov. de Malleco, Termas de Río Blanco, Cautín.

\section{Época de vuelo: Febrero.}

Material examinado: (9 machos, 2 hembras): 1 macho Holotipo, Termas de Río Blanco,
Cautín, ii-51 (gen. prep.) (UCCC); 1 hembra, Alotipo, Termas de Río Blanco, Cautín, ii-51 (gen. prep.) (UCCC); Paratipos: 8 machos, Termas de Río Blanco, Cautín, ii-51 (UCCC); 1 hembra Paratipo, Termas de Río Blanco, Cautín, ii-51 (UCCC)

Expansión alar: $\mathrm{X}$ macho $=32.4 \mathrm{~mm}(\mathrm{n}=9) ; \mathrm{X}$ hembra $=31.5 \mathrm{~mm}(\mathrm{n}=2)$.

Etimología: dedicada al especialista en Noctuidae de Argentina, Pablo Koehler.

Observaciones: se diferencia de la especie $A$. excellens, en que $A$. paulokeleri presenta una ampulla terminada en punta, alargada que alcanza el borde de la valva, una y media vez el ancho máximo de la valva y $A$. excellens, su largo es un tercio el ancho máximo de la valva.

Argyrana bimorpha n. sp.

(Figs. 4-5, 8 y 10)

\section{ENGLISH DIAGNOSIS OF NEW TAXON}

Diagnosis: morph 1: forewings gray with black scales mixed, in the middle one black band along the base of wings to costal termen, subterminal band with black scale, dentate, tegulae and patagiae gray mixed with piliform black scales, eyes ciliate covered around its. hindwing whitish mixed with brownish scales, lunulae with black scales; morph 2: forewings gray and brownish mixed, subterminal band well-defined, dentate with black scale, patagiae and tegulae with piliform, whithish and black scale mixed, hindwing whitish, lunula spot with black scale. Genitalia: short valva, rounded, twice the maxim wide, uncus rounded with apical spine, ampulla pointed, no reach the costal border triangulate saccus, cornuti with nine spine of bulbous base; spine fine present, long and thin.

Macho (Fig. 4): morfo 1 alas anteriores grises con escamas negras entremezcladas, en el medio del ala una banda longitudinal con 


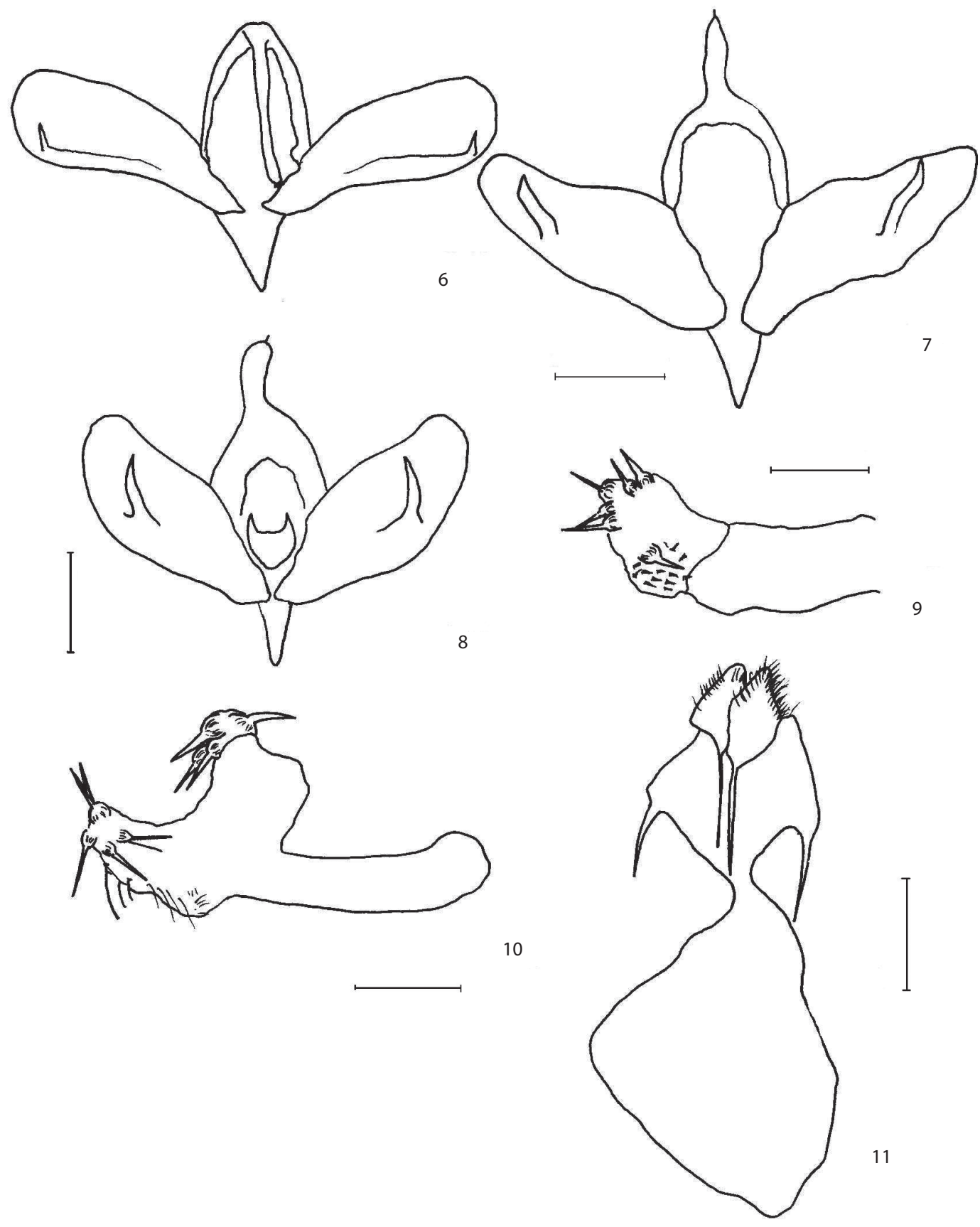

Genitales masculinos: Fig. 6 A. excellens; Fig. 7. A. paulokeleri; Fig. 8. A. bimorpha; Fig. 9 aedeagus A. paulokeleri; Fig 10. aedeagus $A$. bimorpha. Genitalia hembra: Fig. 11: A. paulokehleri.

Male genitalia: Fig. 6 A. excellens; Fig. 7. A. paulokeleri; Fig. 8. A. bimorpha; Fig. 9 aedeagus A. paulokeleri; Fig 10. aedeagus A. bimorpha. Female genitalia Fig. 11: A. paulokehleri. 
escamas negras que atraviesa el ala hasta el borde costal llegando hasta la banda subterminal que se destaca porque esta delimitada por escamas negras, dentada; patagias, tégulas grises con escamas piliformes negras entremezcladas, cilias largas que cubren el ojo; alas posteriores blanquizcas con una lúnula con escamas negras bien notoria, patas con escamas piliformes blanquizcas y escamas castaño-claras entremezcladas. Morfo 2 (Fig. 5): alas anteriores grises con escamas castaño-claras entremezcladas, banda subterminal notoria, dentada, delimitada con escamas negras, patágias, tégulas con escamas piliformes blanquizcas y negras entremezcladas, patas con escamas piliformes blanquizcas y castaño-claras, alas posteriores blanquizcas con una lúnula con escamas negras bien notorias.

Genitales masculinos (Figs. 8 y 10): valvas cortas, redondeadas, ancho máximo dos veces el largo de la valva, uncus redondeado terminado en una espina, presencia de ampulla terminada en punta, no alcanza el borde costal de la valva, saccus triangular, cornuti con nueve espinas de base bulbosa, presencia de espinas finas, largas y delgadas en número indeterminado.

Hembra: no se conoce.

Distribución geográfica: Chile: Aysén.

Época de vuelo: Octubre (UCCC data).

Material examinado: (11 machos): 1 macho, Holotipo, Chile, XI Región, Región de Aysén, matorral 3, 10-11-octubre-2006, 666023, 4739505, 379 m.s.n.m. (A)(gen. prep.); Paratipos: 1 macho, Chile, XI Región, Región de Aysén, matorral 3, 10-11-octubre-2006, 666023, 4739505, 379 m.s.n.m. (X) (gen. prep.) 3 macho, Chile, XI Región, Región de Aysén, matorral 8, 13-14-octubre-2006, 644358, 4715278, 29 m.s.n.m. (B) (gen. prep); 3 machos, Chile, XI Región, Región de Aysén, matorral 8, 13-14-octubre-2006, 644358, 4715278, 29 m.s.n.m.; 1 macho, Chile, XI región, renoval 5, 648345, 471792, 45 m.s.n.m. T-162; 2 machos
Chile, XI Región, Aysén, renoval 6, 13-14-octubre-2006, 640742, 4715480, 27 m.s.n.m.

Expansión alar: X machos: $32.5 \mathrm{~mm}(\mathrm{n}=9)$

Etimología: se alude a los dos morfos cromáticos de esta especie.

Observaciones: se asemeja a la especie $A$. paulokeleri en la forma de la valva, la ampulla terminada en punta, saccus triangular, ausencia de corona, pero se diferencia en que la ampulla no alcanza el borde costal de la valva, el aedeagus de $A$. bimorpha presenta nueve espinas de base bulbosa y A. paulokeleri presenta seis espinas de base.

\section{DISCUSIÓN}

El género Argyrana Koehler, es semejante al nuevo género Gentiliana (Olivares \& Angulo in litt.), con el cual comparten la ausencia de corona, ampulla única, largo de la ampulla dos veces el largo de la valva, saccus alargado, pero se diferencian en que la ampulla de Argyrana es lisa, no dentada como en el nuevo género, aedeagus con un cornuti con espinas de base bulbosa y hembra con una bursa copulatrix unisacular.

La época de vuelo del género, que corresponde a noviembre (Koehler 1951), se amplía a octubre y febrero en el presente trabajo.

El género se registra por primera vez en Chile, ya que ya había sido citado para Argentina (Santa Cruz, Comodoro Rivadavia y Chubut) (op. cit.).

\section{AGRADECIMIENTOS}

Deseamos agradecer a Fernando Navarro y Adriana Chalup por el apoyo logístico durante nuestra estadía en el Instituto Miguel Lillo para la revisión de la Colección Koehler, también agradecemos al proyecto $\mathrm{N}^{\mathrm{o}}$ 207.113.074-1.0 de la Dirección de Investigación de la Universidad de Concepción por el apoyo financiero a la presente publicación. 


\section{RESUMEN}

El género de la polilla Argyrana fue descrito por Koehler en 1951. Se estudiaron 539 especímenes y aquí se redescriben los adultos, los órganos genitales del macho y la hembra. Se reportan dos nuevas especies para la Araucanía y Aysén (IX Región y XI Región, Chile; el género era previamente conocido sólo para Argentina). Estas polillas vuelan en Octubre, Noviembre y Febrero.

Palabras clave: Lepidoptera, Argyrana, Cucullinae, nuevas especies, andino-patagónicos.

\section{REFERENCIAS}

Angulo, A.O. \& G.T. Weigert. 1977. Pseudaletia punctulata (Blanchard) y Pseudaletia impuncta (Guenée): nóctuidos hadeninos similares en Chile. Lepidoptera: Noctuidae). AgroSur 5: 12-17.

Koehler, P. 1951. Las Noctuidae Argentinas. Subfamilia Cucullianae. Acta Zool. Lilloana 12: 135-181.

Poole, R.W. 1989. Lepidopterorum Catalogus (N.S.) Fascicle 118. Noctuidae. Part. 1. E.J. Brill/Flora and Fauna Publications, Florida, EEUU. 
